\title{
Estrategia de prevención de enfermedades relacionadas con virus papiloma humano. Fundamentación de una decisión programática en Chile
}

\author{
Gabriela Moreno \\ Strategy for the prevention of papillomavirus related diseases. \\ Substantiation of a programmatic decision in Chile
}

Réplica a Punto de Vista de A. Fica (pág. 196)

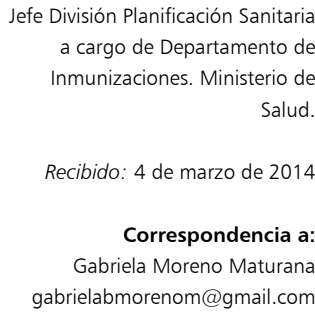

gabrielabmorenom@gmail.com

\section{Sr Editor:}

En relación con el artículo de Alberto Fica publicado en este número de la revista (págs.: 196-203), que se refiere a la incorporación de la vacuna contra la infección por el virus papiloma humano (VPH) al Programa Nacional de Inmunizaciones, como Ministerio de Salud (MINSAL) y con la responsabilidad de liderar la División de Planificación Sanitaria, en la que está el Departamento de Inmunizaciones, quiero compartir con la comunidad científica algunos de los aspectos considerados en la incorporación de esta vacuna.

Dicha infección es un problema de salud pública en el espectro completo de los efectos que causa, que van desde verrugas genitales, papilomatosis respiratoria, al cáncer cérvico-uterino e incluyendo otros tipos de cánceres asociados al VPH. Es por ello que el calendario oficial de inmunizaciones 2014 indica "vacuna contra virus papiloma humano para prevenir la infección por VPH". Para respaldar esta decisión se ha analizado extensamente la información disponible y las experiencias internacionales, y se ha consultado a distintas sociedades científicas y al Panel de Expertos CAVEI (Comité Asesor en Vacunas y Estrategias de Inmunización).

Con estos antecedentes, el Departamento de Inmunizaciones propuso a la autoridad ministerial el introducir la vacuna tetravalente en las niñas de 9 años de edad, aplicándola en los establecimientos educacionales para alcanzar coberturas de $95 \%$. Esto, en el contexto de una estrategia que considere no sólo la intervención de vacuna sino que el mejorar la vigilancia de infecciones de transmisión sexual en centros centinela, las coberturas de tamizaje con Papanicolau y/u otras técnicas de diagnóstico precoz $y$, en especial, vigilar la inmunogenicidad de la vacuna aplicada en nuestra población para evaluar la evolución de los niveles de anticuerpos en el tiempo.

Algunos aspectos relevantes de los análisis indican que la infección por VPH se ha asociado con afecciones no cancerosas, tanto en hombres como mujeres y, además del cáncer de cuello uterino, con otros tipos de cáncer, la mayoría potencialmente prevenibles a través del uso de vacunas contra el VPH. Entre las condiciones relacionadas con VPH prevenibles no cancerosas, las verrugas genitales y la papilomatosis respiratoria están inequívocamente vinculadas a los VPH tipos 6 y 11 . En los cánceres vaginales y sus lesiones precursoras se detecta ADN de VPH en la mayoría de los casos; en estudios recientes, se reportó ADN de VPH positivo en 64 a $91 \%$ de los casos de cáncer vaginal y 82 a 100\% de las neoplasias intraepiteliales vaginales de grado 3 (VAIN3). Se estima que 40 a $50 \%$ de los cánceres de vulva también se asocian con VPH. En los hombres, el ADN de VPH se encuentra regularmente en los cánceres de pene (40-45\%). En ambos sexos, el ADN del VPH se detecta en la mayoría de los cánceres anales (88-94\%). En los cánceres de cabeza y cuello, la prevalencia de ADN de VPH varía mucho según el estudio y la geografía. Una relación consistente con VPH se ha observado en los cánceres de la orofaringe, encontrándose el ADN del VPH en 35 a 50\% de los casos observados en países desarrollados. En todos los tipos de cáncer VPH positivos no cervical, el VPH 16 es el tipo más común detectado, seguido por los VPH 18, 31, o bien 33 y 45. Los estudios sobre la incidencia del cáncer de cuello de útero han conducido a la idea de que esta patología es la que domina la carga de enfermedad relacionada con el VPH. Como consecuencia, ese fue el modelo para los estudios de vacunas, el desarrollo de estrategias y políticas de inmunización, los modelos económicos sanitarios y el desarrollo de estrategias de comunicación en el control del VPH. La historia natural y la epidemiología de otros cánceres relacionados con el VPH han sido mucho menos conocidas pero, en los últimos años, nueva información proveniente de distintas fuentes ha ampliado nuestro conocimiento de las enfermedades relacionadas con el VPH, y por tanto nos obliga a repensar la estrategia de control que hasta ahora ha sido principalmente orientada 
Carga de enfermedad en ambos géneros

Eficacia vacuna en hombres

Accesibilidad de vacunas

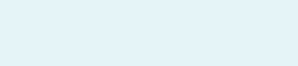

Estudios clínicos sobresalientes
La etiología viral (VPH) ha sido establecida para una fracción significativa de cánceres de vulva, vagina y ano, en ambos géneros, y pene. Una fracción significativa de cáncer oro-faríngeo en ambos géneros, está altamente asociada con infección por VPH. Los datos de países industrializados con programas de tamizaje sugieren que el número de casos de cáncer anal y oro-faríngeo está aumentando y puede haber sobrepasado el cáncer cervical. Ha habido un incremento del conocimiento de la co-morbilidad entre VPH y virus de inmunodeficiencia humana (VIH), las mayores tasas de mortalidad tienden a ocurrir en los mismos países (África sub-sahariana)

Altas tasas de cáncer anal en hombres que tienen sexo con hombres y en individuos VIH (+)

Datos iniciales en Australia están demostrando alta eficacia en la prevención de verrugas genitales en cohortes de niñas vacunadas, y eficacia significativa, aunque menor, en hombres no inmunizados de la misma población, ejemplo significativo de impacto de la inmunidad de rebaño Datos de los estudios en hombres han demostrado alta eficacia contra lesiones anales pre-cancerosas relacionadas con VPH y cáncer anal y alta eficacia en evitar verrugas genitales

La Alianza GAVI (Global Alliance for Vaccines and Immunisation) ha incluido a las vacunas contra VPH en la lista de vacunas que deben ser apoyadas financieramente en países elegibles abriendo la posibilidad de incluirla en países en vías de desarrollo. El precio de la vacuna contra VPH tetravalente está siendo ofrecida a $U \$ 5 /$ dosis. Muchos otros organismos multinacionales (OPS, Fondo rotatorio) y oficinas nacionales han negociado precios que facilitan el desarrollo de programas de vacunación pública

Se han hecho análisis económicos para reflejar estos niveles de precios desde que los países en desarrollo deben co pagar una fracción del precio.

En el largo plazo, agregar vacunas contra VPH en los calendarios de vacunación del lactante disminuiría los costos de entrega y permitiría que esté disponible para el $80 \%$ de los niños del mundo. Aun si el refuerzo fuera necesario en pre-adolescentes, la estrategia sería altamente exitosa Los fabricantes necesitarían estudios puentes que se pueden realizar en grupos relativamente pequeños

Estudios con nuevas vacunas contra VPH están en ejecución, productos que incluyen tipos adicionales de VPH. Si son exitosos, esas vacunas modificarán dramáticamente la estrategia de prevención, las poblaciones objetivo y los protocolos de tamizaje en mujeres vacunadas

Adaptado de ref. 3 al cáncer del cuello del útero. Bosch y cols., realizaron un análisis de la nueva información y resume los principales elementos que impulsan el cambio del paradigma en la prevención de la infección asociada al VPH (Tabla 1).

Cuando los países evalúan incorporar una vacuna contra VPH en los programas nacionales, una categoría importante en la evaluación es la comparación de la relación costo-efectividad de las vacunas bivalentes y tetravalentes. En general, tal comparación depende de un complejo equilibrio entre los supuestos sobre duración de la protección directa, el alcance y la duración de la protección cruzada, y la protección contra verrugas anogenitales y papilomatosis respiratoria recurrente. En este contexto, una ventaja de la vacuna tetravalente es los beneficios adicionales y ahorros de costos asociados a la protección contra las verrugas ano-genitales conferida por la inclusión de los tipos de VPH 6 y 11. Si las diferencias potenciales de protección cruzada o los precios de las vacunas no se consideran, varios estudios han encontrado que la relación costo-efectividad de la vacunación contra el VPH 16, 18, 6 y 11 es más favorable que para los VPH 16 y 18 por sí solos.

En los análisis de costo-efectividad de las vacunas generalmente se asume, en el caso base, que ambas vacunas tendrán muy larga duración o protección de por vida para los tipos de VPH incluidos en las vacunas. Los resultados clínicos de los ensayos de fase 3 han aportado datos de seguimiento cercano a 10 años, una meseta en los títulos de anticuerpos contra VPH 16 después de varios años de seguimiento sugiere que la protección probablemente se mantenga en el largo plazo; sin embargo, en el caso de la vacuna tetravalente no está claro si la protección será sostenida en el largo plazo para VPH 18. La vacuna bivalente induce altos títulos de anticuerpos neutralizantes en suero; sus implicancias clínicas a largo plazo son desconocidas, es posible que eventualmente se asocie con una mayor duración de la protección. Los resultados de ensayos de ambas vacunas sugieren que la vacuna bivalente puede estar asociada con mayor nivel de protección cruzada contra tipos no incluidos en la vacuna, pero las diferencias en los métodos de análisis y los tiempos de seguimiento hacen que sea difícil comparar directamente los resultados. La duración de la protección cruzada es también un área de incertidumbre dado que los títulos de anticuerpos neutralizantes son sustancialmente más bajos para los tipos no incluidos en la vacuna.

Un análisis comparativo a un precio equivalente en tres países llegó a la conclusión de que una vacuna bivalente se convierte en rentable si proporciona 22 a $44 \%$ más de protección cruzada contra tipos no incluidos en la vacuna, que una vacuna tetravalente. Un análisis en Italia concluyó que los ahorros de costos por verrugas ano-genitales podrían ser compensados por los ahorros adicionales en costos de tratamiento de las lesiones pre-cancerosas y de cáncer de cuello uterino que se derivan de una mayor protección cruzada por la vacuna bivalente. Sin embargo, si se tiene en cuenta aspectos de calidad de vida, la vacuna tetravalente podría ser más atractiva debido a la ganancia que representa la prevención de verrugas ano-genitales.

Un enfoque alternativo es calcular el costo umbral en el que las dos vacunas tienen rentabilidad equivalente. Jit y cols., han realizado un análisis en el Reino Unido, que considera una serie de resultados, la duración de la protección y protección cruzada, y concluyó que la 
vacuna bivalente tendría que ser 19-35 libras esterlinas (aproximadamente 22 a 41\%) menos costosa por dosis para tener rentabilidad equivalente a la vacuna tetravalente, principalmente debido a la falta de protección contra las verrugas ano-genitales. Este hallazgo es consistente con un análisis para Irlanda y Canadá en que la vacuna bivalente tendría que ser 22 a 26\% menos costosa, aunque estos análisis consideran sólo los resultados de cáncer cérvico-uterino y verrugas ano-genitales. Esto implica que la diferencia en la rentabilidad de las vacunas está dada principalmente por la carga de enfermedad de las verrugas ano-genitales, los costos relativos del tratamiento de las verrugas, lesiones pre-cancerosas, y el impacto asociado a la calidad de vida.

Varios países han incorporado vacuna tetravalente en sus programas de inmunización con diferentes coberturas de vacunación. El primer estudio analizado es la experiencia de California. Se estima que 1,4 millones de estadounidenses tienen verrugas genitales. La vacuna cuadrivalente está disponible en los Estados Unidos de América (E.U.A.) desde junio de 2006. El Comité Asesor sobre Prácticas de Inmunización (ACIP) recomienda la vacunación de rutina de niñas y niños a los 11 a 12 años y vacunación de puesta al día ("catch up") para las niñas de 13 años y niños de 13 a 21 años. Desde el año 2010, $49 \%$ de las adolescentes entre 13 y 17 años en E.U.A. ha recibido al menos una dosis y $32 \%$ de ellas ha recibido las tres dosis establecidas en el protocolo; $21 \%$ de las mujeres de 19 a 26 años ha recibido al menos una dosis y en California, 56\% recibió al menos una dosis de vacuna. Hay varios estudios en marcha para medir el impacto con base poblacional de la vacunación. Se ha planteado que dada la rápida evolución de las verrugas genitales después de la infección, el seguimiento de la tendencia de estas lesiones puede proporcionar las primeras pruebas de efectividad de la vacuna.

Entre los años 2007 y 2010, un promedio de 1.754 .000 mujeres y 258.000 hombres han sido vacunados anual- mente, cifras que aumentaron cada año en casi todos los grupos de edad. En general, 0,7\% de las mujeres y 3,3\% de los varones fueron diagnosticados con verruga genital. Las mayores tasas se presentaron entre los 21 a 25 años, mientras que las más bajas estaban en los mayores de 30 años. Entre 2007 y 2010, el diagnóstico de verruga genital disminuyó en 34,8\% (IC 95\% = -38,2-31,5\%) entre mujeres menores de 21 años, desde $0,94 \%$ a $0,61 \%$ $\left(\mathrm{P}_{\text {trend }}<0,001\right)$.

En el año 2007, Australia se convirtió en uno de los primeros países en poner en práctica un programa nacional de inmunización con vacuna tetravalente, a las niñas de 12 a 13 años de edad en las escuelas. Desde 2007 a 2009 se implementaron dos programas de puesta al día, uno para niñas de 13 a 18 años en las escuelas y otro para mujeres de 18 a 26 años de la comunidad. En el año 2010, las tasas de cobertura de vacunación del programa basado en la escuela fue $83 \%$ para la primera dosis, $80 \%$ para la segunda dosis y $73 \%$ para la tercera dosis, con tasas de cobertura que disminuyeron al aumentar la edad. Se estableció una red de vigilancia centinela para vigilar el efecto de la vacuna sobre los casos de verruga genital a través de Australia. Datos iniciales mostraron que, dos años después de la introducción de la vacuna, la proporción de verrugas genitales diagnosticada se redujo en 59\% en las mujeres de 12 a 26 años elegibles para vacunar en 2007 y en $39 \%$ en los hombres heterosexuales de la misma edad. La disminución de las verrugas genitales en hombres heterosexuales de grupos más jóvenes puede ser atribuida a la inmunidad de rebaño, en otras palabras, la protección indirecta de personas no vacunadas como resultado de la reducción de la exposición a la infección.

Dada esta información sabemos que hay impacto a corto plazo que no debemos eludir y que quedan interrogantes para los efectos a largo plazo que se aclararán a medida que los países ganemos evidencia epidemiológica que permita mejorar la gestión de los programas y adecuar las políticas preventivas de salud

\section{Referencias bibliográficas}

1.- Giuliano A R, Tortolero-Luna G, Ferrer E, Burchell A N, de Sanjose S, Kjaer S K, et al. Epidemiology of human papillomavirus infection in men, cancers other than cervical and benign conditions. Vaccine 2008; 26 (Suppl 10): K17-28.

2.- Backes D M, Kurman R J, Pimenta J M, Smith J S. Systematic review of human papillomavirus prevalence in invasive penile cancer. Cancer Causes Control 2009; 20 (4): 449-57.

3.- Bosch X, Tsu V, Vorsters A, Van Damme P, Kane M. Reframing cervical cancer prevention. Expanding the field towards prevention of human papillomavirus infections and related diseases. Vaccine 30S (2012): F1-F11.

4.- Canfell K, Chesson H, Kulasingam S, Berkhof J, Díaz M, Kim J. Modeling preventative strategies against human papillomavirus-related disease in developed countries. Vaccine 30S (2012): F157-F167.

5.- Jit M, Chapman R, Hughes O, Choi Y H. Comparing bivalent and quadrivalent human papillomavirus vaccines: economic evaluation based on transmission model. Br Med J 2011; 343: d5775.

6.- Dee A, Howell F. A cost-utility analysis of adding a bivalent or quadrivalent HPV vaccine to the Irish cervical screening programme. Eur J Public Health 2010; 20 (2): 213-9.
7.- Brisson M, Van de Velde N, De Wals P, Boily M C. The potential cost effectiveness of prophylactic human papillomavirus vaccines in Canada. Vaccine 2007; 25 (29): 5399-408.

8.- Bauer H, Wright G, Chow J. Evidence of human papillomavirus vaccine effectiveness in reducing genital warts: an analysis of California public family planning administrative claims data, 2007-2010. Am J Public Health. 2012; 102: $833-5$.

9.- Ali H, Donovan B, Wand H, Read T, Regan D, Grulich A, et al. Genital warts in young Australians five years into national human papillomavirus vaccination programme: national surveillance data. Br Med J 2013; 18: 346: f2032. 